\title{
A Lightweight Wireless Overpressure Node based Efficient Monitoring for Shock Waves
}

\author{
Shang Gao, IEEE Member, Guiyun Tian, IEEE Senior Member, Xuewu Dai, IEEE Senior Member, \\ Qing Zhang, Zhiling Wang, Xinge Yang, Qiaomu Wang, Naishu Jia
}

\begin{abstract}
Overpressure measurement is an important approach to evaluate the power of shock wave (SW) monitoring. Traditional wired monitoring systems exhibit the limitations of high-cost, heavyweight, troublesome maintenance, and big-data transmission in SW monitoring. In this paper, a new lightweight FPGA-based Wireless Overpressure Node (LFWON) with the resistance to high-temperature and high-pressure environment for SW monitoring. The proposed LFWON is based on the Spartan-6 XC6SLX9-2TQG144C FPGA circuit, via a Serial Peripheral Interface (SPI) to the RF transceiver and data bus to the NAND flash chip for data management. To validate the LFWON, experimental tests in terms of dynamic parameters and network quality are performed in a real blast testing with $8 \mathrm{~kg}$ trinitrotoluene (TNT). This paper is conducted to provide new insights into how the anti-shocking structure and sensing algorithm of wireless sensor node is designed in SW monitoring for acquiring overpressure accurately. The results show that the errors of $\Delta P(7 m-12 m), t_{d}(>6 m)$, and $I^{+}(3 m-24 m)$ from proposed LFWON are below $20 \%$ in comparison with wired system. In addition, the RSSI value of LFWON should be set above $-70 \mathrm{dBm}$ for stable communication quality.
\end{abstract}

Index Terms-Overpressure, wireless sensor network, SW monitoring, dynamic parameter, network quality

\section{INTRODUCTION}

$\mathrm{I}_{\mathrm{i}}^{\mathrm{N}}$ $\mathrm{N}$ the recent years, the utilization of explosive weapons[1] including land mines, rocket-propelled grenades (RPGs) and improvised explosive devices (IEDs) increasingly contributes to mild traumatic brain injury (mTBI) frequently or even fatal injury by the overpressure wave coupling with a large amount of energy[2-4]. Thus, the SW monitoring with high-precision and ease-deployed sensors and measurement is required in air explosions for defense and civil applications[5].

The temporal pressure characteristics of SWs including peak pressure, energy, impulse, and the duration of the positive phase $[6,7]$ are employed to estimate explosion power and shock damage. However, it is not easy to measure these dynamic parameters accurately, due to the SW characteristics of complicated blast environment (e.g. short durations, steep rising edges, and extreme conditions over 1000 Celsius degrees along with intense light, reflected pressure, vibrations, ionized gazes)[8]. In most scenarios, accurate experimental data and the

This work was supported in part by Nanjing University of Science and Technology under Research Start-Up Funds under Grant AE89991/032, in part by the Fundamental Research Funds for the Central Universities under Grant 309181A8804 and Grant 30919011263, in part by Natural Science Foundation of Jiangsu Province, China under Grant BK20190464, in part by Jiangsu Planned Projects for Postdoctoral analytical predictive theory models or numerical simulations of parameter measurement [9] jointly determines the accuracy of blast damage effectiveness. In a real experiment, the piezoelectric pressure transducer is selected frequently for explosion tests due to their good sensing performances, wide operating bandwidth, and impact resistance [10-12]. Particularly, to relieve the parasitic effects from thermal shocks, mechanical shocks, and vibrations [13, 14], an additional lightweight mechanical structure for the pressure sensor fixture should be designed.

In SW measurement, the present cables demand that monitoring structure should be piled into the land and a trench is dug for wire running which is also buried into the ground for connecting monitoring points to the center. It is obvious that this kind of cable deployment is timeconsuming and labor-extensive [15]. For example, one monitoring point will produce 4 wires at least and extends over 120 miles of cables. An alternative approach of wireless sensor networks may contribute to $70 \%$ wire reduction, 5 hours saving, and also increase communication range by around $30 \%$. In contrast, a large number of wired centralized sensors connecting with the acquisition unit are managed by long cables, easily leading to operational problems or electrical failures such as short-circuits or electrical breakdowns before the experiment process, further making an adverse impact on the reliability and the accuracy of the overpressure measurement[16]. Additionally, to get a better assessment of brisance, the distributed monitoring management outperforming centralized sensors deployment has become more laborious in SW monitoring.

To address the limitation of cable-cased monitoring systems, the WSN-based measurement such as 4G, 5G, Zigbee, Bluetooth, and other WSN communication has become a new approach to be applied in many new areas [17-20]. However, the $4 \mathrm{G}, 5 \mathrm{G}$ depending on cellular communication contributes to a large amount of energy consumption and the extra increase in operating costs. Especially, the wildest environment of SW monitoring cannot support $4 \mathrm{G}$ and $5 \mathrm{G}$ communication. In addition, ZigBee and Bluetooth based on $2.4 \mathrm{GHz}$ short-range communication which is not meeting for long-distance communication demanded in large-scale SW monitoring. As

Research Funds under Grant 1003-YBA20012, in part by Chinese Postdoctoral Science Foundation under Grant 2020M671481, supported by the National Natural Science Foundation of China under Grant 61527803, 51905242, 61960206010 and 61903193. (Corresponding author: Shang Gao) 
for SW monitoring, some wireless Polyvinylidene Fluoride (PVDF) sensors or surface acoustic wave (SAW) sensors have been developed[21-23]. However, the performance of the PVDF sensor is much worse than the piezoelectric pressure sensor in SW monitoring. Till now, a few pieces of literature have made some good progress on wireless overpressure node design for large-scale SW monitoring. It is proved that Sub$1 \mathrm{GHz}$ has more advantages regarding ultra-long transmission, high stability, ultralow-power, and low cost [24], which offers a new application opportunity for air blast monitoring. Furthermore, with the recent advances in wireless sensor networks (WSN) technology and the Internet of things(IoT), wireless SW systems have emerged as a promising alternative solution for large-scale air blast monitoring, which is beyond the capability of the traditional wired monitoring system.

In this paper, the main contribution is that this is a first attempt to propose a new lightweight underground FPGA-based wireless overpressure node with the resistance to hightemperature and high-pressure underground deploying structure for piezoelectric overpressure sensing. We provide new insight into how a wireless overpressure node is designed for carrying out remote network communication efficiently in blast wave monitoring.

The article is organized as follows. The wireless overpressure-monitoring network design method, the installation of the piezoelectric pressure transducer, the mechanical model of the piezoelectric pressure transducer, and the fixture physical structure of LFWON are presented in Section II. In addition, the system design approach of the LFWON for SW monitoring are also proposed in Section III. In order to verify the structural and sensing performance of LFWON, field experiments in regards to dynamic parameters evaluation and wireless transmission quality are implemented in the air blast field test in Section IV. Lastly, we conclude the work and discuss future work in Section V.

\section{The Integration of LFWON For Air Blast Monitoring}

\section{A. The Installation of Piezoelectric Pressure Transducer on LFWON}

In air blast monitoring, mechanical impact, or vibration, thermal shock results in the parasitic effects of piezoelectric pressure sensors. As for mechanical vibration, it originates from three parts: 1) seismic waves propagating along geological media resulting from explosions; 2) vibration imposed on mounting base of sensor triggered by SW; 3) vibration imposed on sensor triggered by SW. All these parasitic effects influence the output of piezoelectric pressure sensors, even leading to the misreading of pressure and other parameters. Thus, it is necessary to design equipment with a fixture sensor for hindering the parasitic effects.

The piezoelectric pressure sensors are widely used in SW measurement due to their good dynamic characteristics and impact resistance. They have low output impedance, allowing transmission in harsh environments without compromising signal quality. A low-noise pressure transducer (Kistler 211B43) requires constant current (4 $m A$ ) and excitation voltage(24 $V$ ). The low-power chip LM134 operating at current levels ranges from $1 \mu A$ to $10 m A$ as the different set by an external resistor provides constant current and excitation voltage for the pressure transducer, as shown in Fig.1. The voltage $(3.7 \mathrm{~V})$ of Li-ion battery in each LFWON should be raised to $24 \mathrm{~V}$ conforming to the excitation voltage of the Integrated Electronics Piezo-Electric (IEPE) sensor.

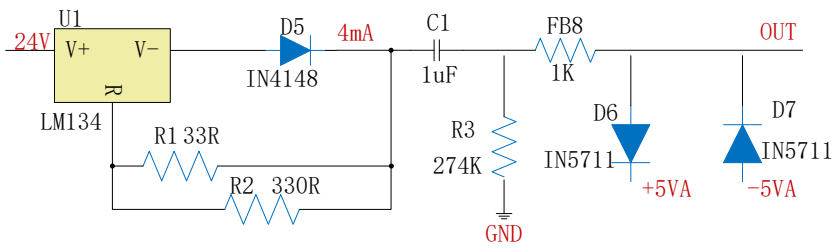

Fig. 1. The conditioning circuit for the piezoelectric pressure transducer The piezoelectric pressure transducer (Kistler 211B43) amounted inside the hole of structure measures transient and repetitive dynamic events in air blast monitoring purposes. It is responsible for converting the charges generated in the transducer into a measurable voltage by an appropriate conditioning electronics. The range of $211 \mathrm{~B} 3$ sensor is $0-500$ psi (34 bar ) and sensitivity is $10 \mathrm{mv} / \mathrm{psi}$. The maximum value supported by the transducer without damage of $211 \mathrm{~B} 3$ is 172 bar. It can also operate in the environment with the temperature ranging from $-54{ }^{\circ} \mathrm{C}$ to $121^{\circ} \mathrm{C}$. The transducer at a given location during an explosion typically presents very fast transient with short rise time $(<<1 u s)$, large bandwidth (between $100 \mathrm{~Hz}$ and $1 \mathrm{MHz}$ ), and high-pressure level $(>10 \mathrm{MPa})$ in the high-temperature environment $(>1000$ ${ }^{\circ} \mathrm{C}$ ). In the air-blast testing, both the wired system and LFWONs aggregate raw overpressure data simultaneously by the same pressure sensor. As shown in Fig.2, the sensor installation on steel structure consists of (1) diaphragm material; (2) wafer material; (3) leg wired of piezoelectric pressure sensor 211B3;

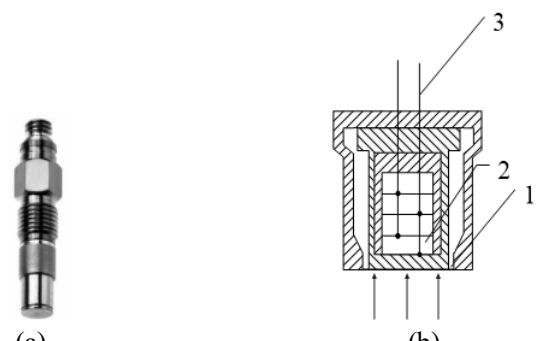

Fig. 2. Piezoelectric pressure sensor $211 \mathrm{~B} 3$ and the installation of the sensor. a) the piezoelectric pressure sensor. b) packaging structure of $211 \mathrm{~B} 3$.

\section{B. The Mechanical Model of Piezoelectric Pressure Transducer}

The integrated circuits piezoelectric (ICP) sensor is commonly used in SW monitoring experiments due to good dynamic characteristic anti-shock ability. The ICP sensor is a diaphragm type structure, which allows the tensioned thinwalled tube to pre-stress the crystal piece. Especially, the external pressure is a very thin diaphragm made of a flexible material. The ICP sensor is considered as a linear time-invariant system, which can be equivalent to a model consisting of a 
piezoelectric element with stiffness $K 1$, a preload spring with stiffness $K 2$, damping $c$, and sensor equivalent mass $m$ according to Fig.2(b). The sensor fixed structure and its equivalent model are shown in Fig.3.

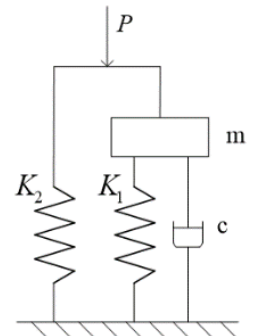

Fig. 3. Equivalent model of diaphragm piezoelectric sensor structure As the pressure on the crystal stack is $P_{1}$ and on the spring is $P_{2}$, the external pressure $P$ is the sum of $P_{1}$ land $P_{2}$. The interactions between $P$ and change $\Delta x$ in compressive deformation of the crystal piece stack is defined as:

$$
P=P_{1}+P_{2}=\left(K_{1}+K_{2}\right) \Delta x
$$

Thus, the effective component of the external force is defined as:

$$
\frac{P_{1}}{P}=\frac{K_{1}}{K_{1}+K_{2}}
$$

In equation (2), the pressure $P_{1}$ received by the crystal stack and is converted to the quantity of electricity after the external pressure $P$ acting on the sensor diaphragm. The final voltage output $U_{0}$ of the sensor is defined as:

$$
U_{0}=\lambda \cdot P_{1}
$$

In equation (3), $\lambda$ is the proportional coefficient between the output voltage and the force of the crystal stack. Thus the sensitivity of the piezoelectric sensor $S$ is calculated as:

$$
S=\frac{U_{0}}{P}=\frac{\lambda \cdot P_{1}}{P}=\frac{\lambda \cdot K_{1}}{K_{1}+K_{2}}
$$

During the SW propagation process, the SW has an impact on the ground and the sensor mounting plate structure. This effect not only produces seismic waves but also generates the vibration of the plate. Seismic wave and mounting plate's vibration have two main impacts on the ICP sensor: First, the plate is subjected to radial stress on the outer wall of the sensor during the vibration process. Secondly, acceleration output during the vibration process will be generated because the piezoelectric crystal has an equivalent mass.

The form effect not only changes the effective component of the force in equation (2) but also changes the stiffness $K 2$ of the preload spring. In this case, the sensitivity of the sensor is the real sensitivity of the sensor. In addition, the crystal stack and the spring are also subjected to the pressure $P^{*}$ due to the prestressing action of the mounting plate. In this case, the amount of compressive deformation on the crystal stack is $\Delta X_{1}$, and then the equation (1) can be translated into:

$$
P+P^{*}=P_{1}+P_{2}=\left(K_{1}+K_{2}\right) \Delta X_{1}
$$

\section{The Fixture Physical Structure of LFWON}

The structure component consists of a pressure sensor, the additional mechanical structure, and M8 screws installing on a test plate. The physical structure of LFWON is a heat annealed copper diaphragm, as shown in Fig.4. It is installed inside the fireball of an explosion for sensing the pressure signal variation closely to the explosive. Groups of LFWONs are located at a different distance away from the TNT sphere. In the experiment, each LFWON is buried into the bunker for measuring reflection pressure. The copper upper and lower layer is $1.15 \mathrm{~mm}$ and $0.85 \mathrm{~mm}$ thick, respectively. Four screw bolts are inserted to connect the upper with lower copper layers together. The material selected for the diaphragm of two layers is copper 12200, heat annealed (at $800{ }^{\circ} \mathrm{C}$ for $1 h$ ), and air-cooled. The exposed area of the upper layer in diameter is mounted by the transducer to monitor overpressure load acting on the outside of the upper layer. Thus, it is required that the copper material should be removed for the transducer. The volume of removal cube in the upper layer has a height of $3 \mathrm{~mm}$ and a diameter of $6.5 \mathrm{~mm}$. To keep the structural integrity of the copper material adjacent to the transducer locations, and avoid the frequency response of the LFWON, the overall height of the transducer is limited to $3 \mathrm{~mm}$. In addition, the transducer width is $6.4 \mathrm{~mm}$ for fitting within $6.5 \mathrm{~mm}$ diameter width blind-hole. The wire inside the LFWON has two ways of transmitting overpressure data by the wired system or wireless system with an antenna. Two holes deployed on opposite and symmetrical sides of LFWON is used for cable extraction and antenna hanged out, respectively, which allows for comparing the measurement results obtained from the wired and wireless systems.
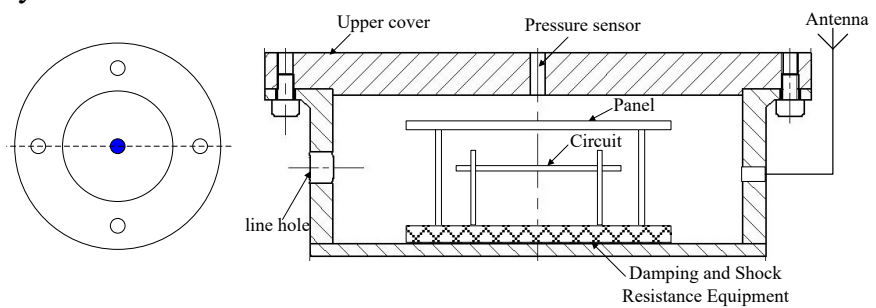

(a)

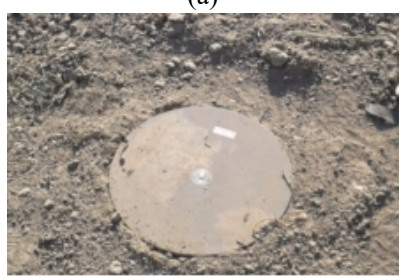

(b)

Fig. 4. The physical structure of LFWON. (a) Sketch of diagram type pressure and packaging structure of LFWON. (b) the wild deployment of LFWON

\section{SySTEM DESIGN OF THE LFWON}

To implement data collection and transmission, we make a detailed design of LFWON which mainly achieves overpressure data collection, data extraction, data temporary storage, LoRa communication, as shown in Fig. 5. The hardware design block diagram is demonstrated in Fig.6. It mainly consists of four parts: Microcontroller Unit (MCU), communication unit, signal conditioning unit, data storage unit, and power supply unit. 


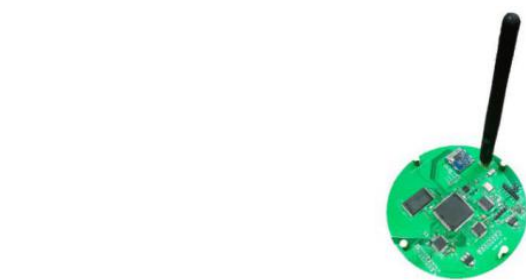

Fig. 5. The hardware entity of LFWON

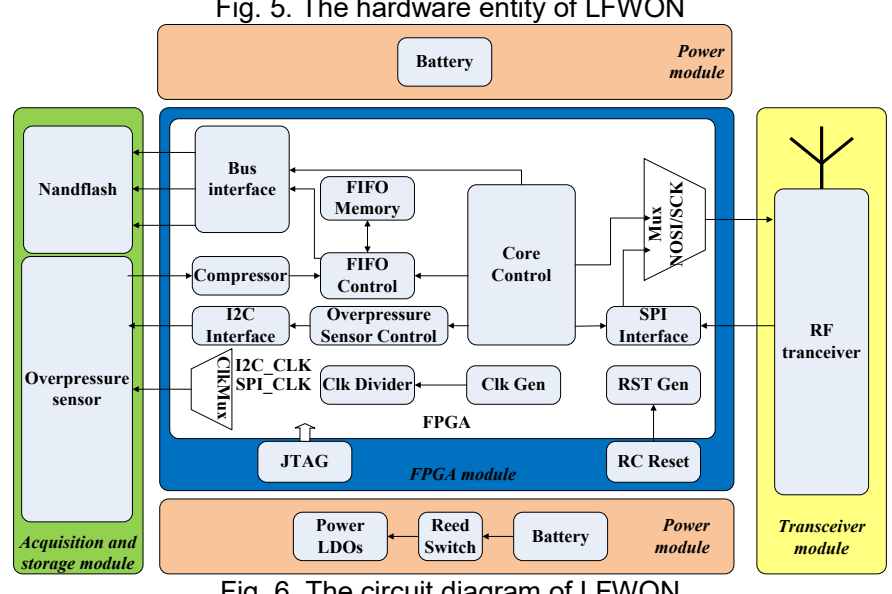

Fig. 6. The circuit diagram of LFWON

\section{A. The FPGA Control Module}

As one of the Spartan-6 series manufactured by Xilinx Company, the MCU of XC6SLX9-2TQG144C running at 100 $\mathrm{MHz}$ is chosen for the LFWON to manage data acquisition, data communication, data extraction, and data storage management. The processor has an optimized 32-bit Reduced Instruction Set Computer (RISC) and Harvard architecture. It integrates with 32-bit general registers, 32-bit instruction word with three operands and two addressing modes, 70 configuration options, and 32-bit address bus. The Linux system can be operated in the processor.

1)The FPGA control module

The control block controls the overall operation of the electronic capsule. As shown in Fig.7, a finite state machine (FSM) is implemented in the control block. The reset signal makes the control block initialize the RF transceiver by SPI interfaces and NandFlash module configuration by interface bus. After that, it flushes the internal first in first out (FIFO) buffer inside both the RF transceiver and NandFlash module and keeps the data acquisition state. Then, the FSM waits for a trigger pulse from a pin connecting to the piezoelectric pressure sensor. If the acquired pressure value of SW exceeds the set threshold inside LFWON, it is indicated that the SW has arrived at LFWON and makes an effect on the piezoelectric pressure transducer. Then, the state machine enters into data acquisition and storing process during several milliseconds. Sequentially, the RF transceiver is set to TX mode and starts to send overpressure data bits to the FIFO control block. The FSM then requests the FIFO control block to send a complete data packet with 256 bit to the RF transceiver directly through the SPI interface. When all storage packets are sent, it stops data acquisition and flashes RF FIFO buffer, sets the RF in RX mode, and starts to wait for the next command.

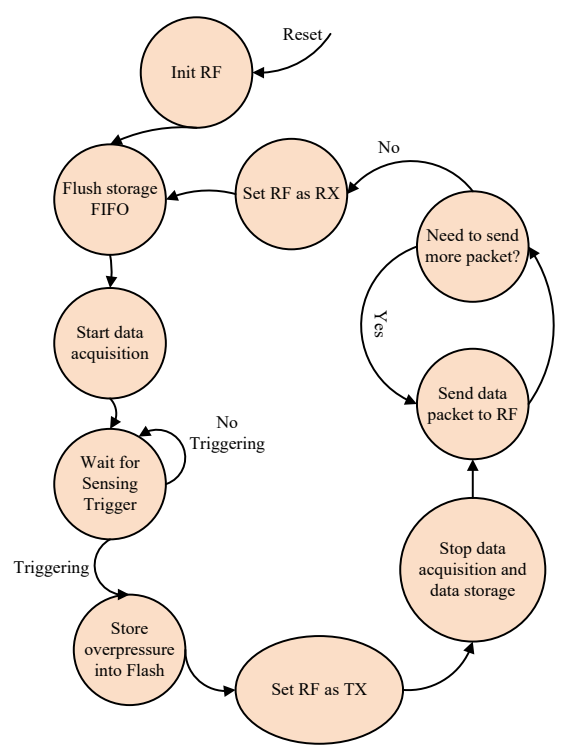

Fig. 7. The FSM flow inside the control block 2) LRU and CS algorithm

The NAND flash memory chip from Samsung Co.Ltd, K9GAG08U0M with the reading or writing sector size of 512 bytes, provides the operation of Least Recently Used (LRU) algorithm and Continuous Stored (CS) algorithm. Before air blast is triggered, the LFWON is required to implement the LRU for waiting for the arrival of SW at the LFWON. As the acquisition value of overpressure exceeds the setting value inside LFWON, the storage mode of LFWON s switches to the CS algorithm from the LRU algorithm. The LRU algorithm is to keep a fixed size of RAM buffer space in Xilinx chip, store inputting data in the RAM buffer in turn, and always keep the latest data at the top, and the earliest data at the bottom of the buffer. When new data are added but the buffer is full, the LRU algorithm substitutes data at the bottom of the buffer, keep the new data at the top and finish data processing of the last element. As shown in Fig.8, the working steps of the LRU algorithm and CS algorithm is as follows:

1) Define and format the RAM in Xilinx chip $(<10 \mathrm{M})$ and large storage space in Nandflash chip $(>1 \mathrm{G})$, point the pointer to the first address of the small storage space.

2) Fill acquisition data into the buffer and the pointer is added automatically.

3) As the pointer points to the last bit of the free storage space and new overpressure data are demanded to be added to space continuously, the pointer points to the first bit of the storage space and the last frame data in the buffer will be eliminated, which is recycled in this way in a given period.

4) As the acquisition value of overpressure exceeds the setting value inside the LFWON, stops storing data into the small storage space and transfer the pointer to the first address of the large storage space for the continuous and successive storing of actual overpressure data. 


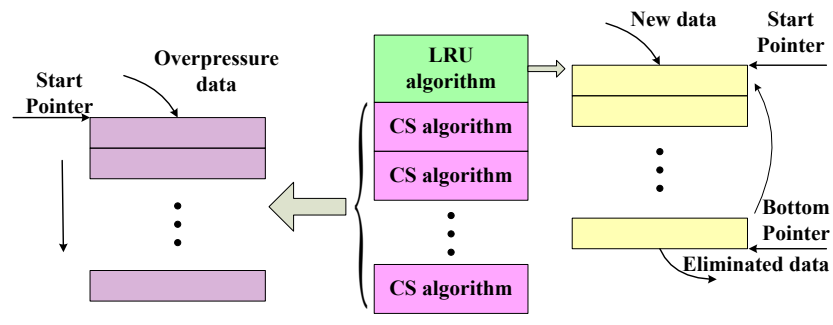

Fig. 8. Working procedure of $L R U$ and $C S$ algorithm

\section{$B$. The power module}

The accumulator is two standard $3.7 \mathrm{~V} \mathrm{Li}$-ion batteries series, which constructs a power package complete with charge, storage, and discharge functions. The DC-DC converters are classified as DC7.4-DC5V, DC7.4-DC3.3V, and DC7.4DC24V converters. As the DC7.4-DC5V converter, the TPS65133 chip with low-voltage characteristics is chosen as a power supply for the LFWON, while AMS1117-3.3 chip is used as DC7.4-DC3.3V converter for the LFWON. Additionally, the TPS61041 chip is dedicated to DC7.4-DC24V for the pressure transducer. To reduce power consumption, a MOS transistor is used to control the power on or off of LFWON to whether collecting data or not.

\section{The transceiver module}

As the communication unit, a low-power RF chip Silicon SI4463 is chosen as a communication unit that is connected to the XC6SLX9 processor through SPI ports for exchanging commands and transmitting wireless data. It integrates an enhanced 8051 processor with a Sub-1GHz RF transceiver working at $433 \mathrm{MHz}$ frequency. The size of the transceiver is $6 \mathrm{~mm} \times 6 \mathrm{~mm} \times 0.85 \mathrm{~mm}$ and it requires a Sub- $1 \mathrm{GHz}$ chip antenna with a dimension of $5.6 \mathrm{~mm} \times 2.1 \mathrm{~mm} \times 1 \mathrm{~mm}$.

\section{$D$. The dynamic parameters measurement principle of LFWON}

The LFWON for the air-blast test is evaluated by the characteristics of the dynamic parameters of SW. The recording data of pressure vs. time history are used to determine the characteristic parameters of the SW (shown in Fig.9) including the time of arrival of the SW $\left(t_{a}\right)$, the peak overpressure maximum $(\Delta P)$, the impulse $\left(I^{+}\right)$, and the duration $\left(t_{d}\right)$ of the positive phase.

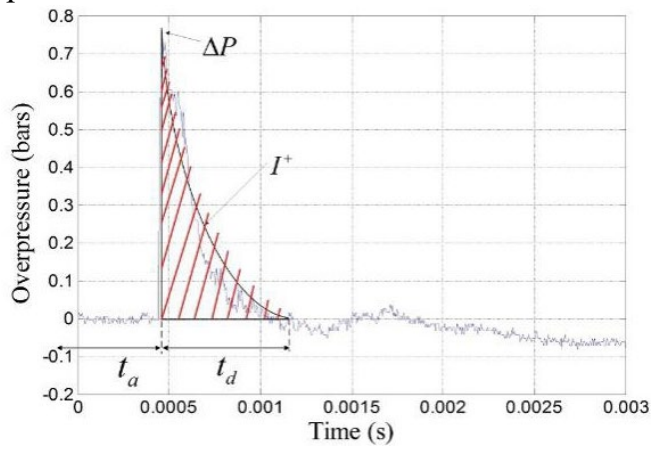

Fig. 9. Definition of the characteristic parameters of the SW

Generally, under the unlimited and Infinite blast boundary, some approaches to evaluate the peak overpressure only relies on the TNT mass $W$ and the distance $R$ between measuring points and the blast center. These approaches have been empirically established to express the maximum overpressure in the field of a strong detonation of explosives. The positive phase of this overpressure signal can be mathematically expressed by a Hoplinson-Cranz model[25, 26] as:

$$
\Delta P=a_{1}\left(\frac{W^{1 / 3}}{R}\right)+a_{2}\left(\frac{W^{1 / 3}}{R}\right)^{2}+a_{3}\left(\frac{W^{1 / 3}}{R}\right)^{3}
$$

where $a_{1}, a_{2}, a_{3}$ are constant values.

Another overpressure expression is defined as:

$$
\Delta P=a \mathrm{e}^{-b\left(\frac{W^{1 / 3}}{R}\right)}
$$

where $a$ and $b$ are constant values.

The duration of the positive phase $t_{d}$ is another important factor to assess the brisance. In respects to engineering theory analysis, the $t_{d}$ is calculated by the M.A.Sadoski model [27]:

$$
t_{d}=c R^{d} W^{e}
$$

where $c, d$ and $e$ are constant values.

The impulse $I^{+}$is equal to the integral of the SW time signal. It is the sum of the positive impulse and the negative impulse, which have the same order of magnitude. According to Kinney's formulas, the impulse of the positive phase depends on the overpressure value $\Delta P$ of the $\mathrm{SW}$ surface and the duration $t_{d}$ of the positive phase[28]. It is defined as

$$
I^{+}=f \frac{W^{2 / 3}}{R}
$$

where $f$ is a constant value.

\section{SySTEM TEST AND ANALYSIS}

To evaluate the structural and system performance of LFWON, the experiments of air-blast field monitoring and wireless quality measurement for the LFWON are carried out.

\section{A. Dynamic parameter evaluation in Air-Blast Testing}

We construct SW monitoring system based on multiple LFWONs which are located as circular distribution at different distances from $3 \mathrm{~m}$ to $31 \mathrm{~m}$ away from the explosive centre, as shown in Fig.10. Groups of LFWONs with power supply arranged into clusters circularly collect overpressure data and conduct communications with the base-station. The LFWON configuration, network communication with time slot assignment, the relaying of commands and data between the LFWONs and the base-station are implemented. The LFWONs are dedicated for signal acquisition, overpressure data processing and transmission, while the base-station has a wired Ethernet interface to the server and is responsible for the establishment, management and maintenance of the monitoring network. 


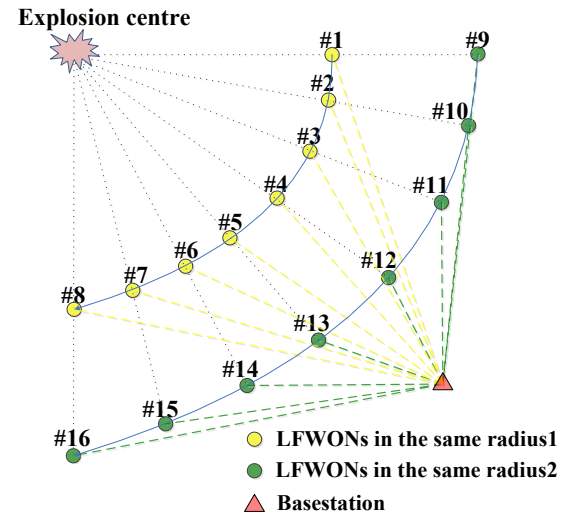

Fig. 10. The diagram of the Wireless overpressure monitoring system

The experimental data are acquired from the detonation of near-field $8 \mathrm{~kg} \quad$ TNT blast. All the LFWONs capture the SW signal and calculate the parameters in light of Section III. Additionally, a wired system (in Fig.11)consisting of a signal conditioning board (Kistler 5148M06), and measurement system (PXIe-1062Q equipped with a high-accuracy acquisition card PXI-5105 and controller PXIe-8135 in Fig.12) has also been equipped in the air-blast testing for data comparison. In respect to the consistency comparison of data measurement, the sampling frequency of both systems is set to the same value of $1 \mathrm{MHz}$ along with sampling length setting to $600 k$. As shown in Fig.13, these final results reveal that the correctness and feasibility of LFWONs for air blast monitoring are verified.

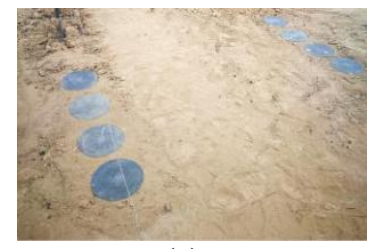

(a)

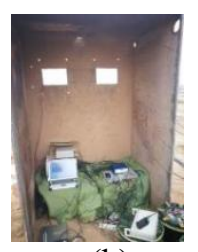

(b)
Fig. 11. A wireless and wired experimental test for air-blast monitoring. (a) the monitoring arrays of LFWONs. (b) wired monitoring system

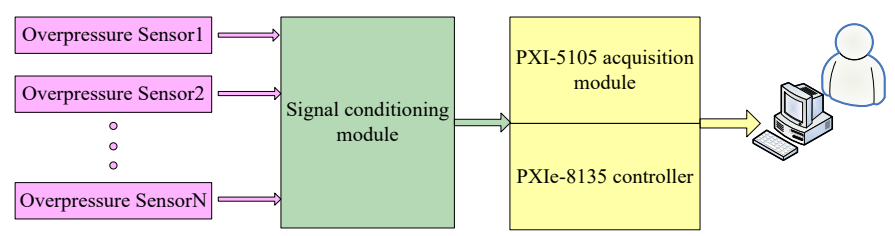

Fig.12 the wired overpressure testing system

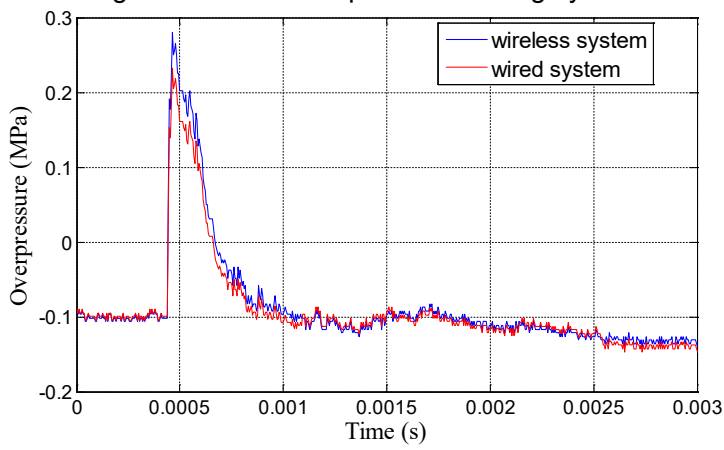

Fig. 13. The overpressure value and the error of overpressure signals between LFWONs and wired system

Before the real experiment evaluation, the diameter effects of the plate on overpressure should be investigated to acquire the best performance of overpressure testing. The interactions between different diameters of LFWON and overpressure peak can be simulated by LS-DYNA software. It can be seen in Table I that the maximum overpressure peak is $0.4932 \mathrm{Mpa}$, as the distance between LFWON and explosion centre is $4 \mathrm{~m}$. The results show that the diameter of plate setting to $20 \mathrm{~cm}$ in experiment evaluation has the best overpressure effects of blast wave.

TABLE I THE SIMULATION FOR THE DIAMETER EFFECT OF
PLATE ON OVERPRESSURE MEASUREMENT
\begin{tabular}{cc} 
The diameter of plate $(\mathrm{cm})$ & Overpressure peak value $(\mathrm{Mpa})$ \\
\hline 10 & 0.2863 \\
20 & 0.4932 \\
24 & 0.4812 \\
26 & 0.4742 \\
28 & 0.4759 \\
32 & 0.4764 \\
34 & 0.4798 \\
36 & 0.4821 \\
38 & 0.4830
\end{tabular}

In this evaluation, the measured maximum overpressure values of LFWON vs. time at different location distances are shown in Fig.14 and Table II. The largest overpressure can reach $0.80480 M p a, 0.13420 M p a, 0.08964$ Mpa , $0.02937 \mathrm{Mpa}$ at the distance of $3 \mathrm{~m}, 6 \mathrm{~m}, 8 \mathrm{~m}, 12 \mathrm{~m}$ respectively. In contrary, the duration of the positive phase $t_{d}$ decreases as the distance increases. On the whole, the impulse $I^{+}$decreases as the distance increases. It is because the energy of SW attenuates as spreading in air.

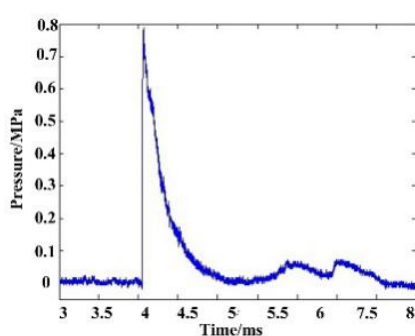

(a)

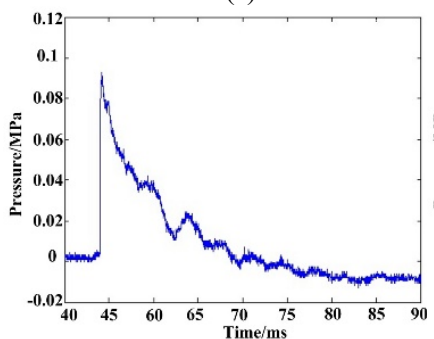

(c)

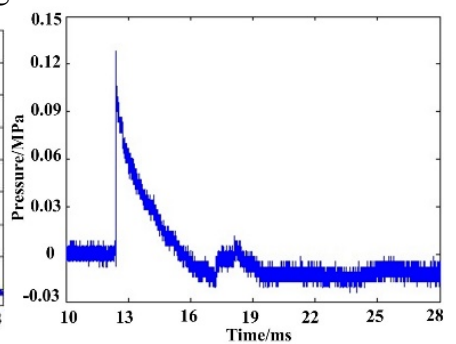

(b)

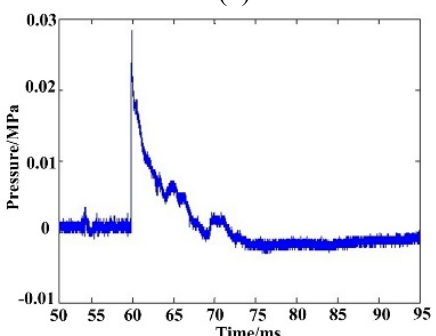

(d)
Fig. 14. The measured overpressure value at different distances in $8 \mathrm{~kg}$ TNT blast. (a) $3 \mathrm{~m}$; (b) $6 \mathrm{~m}$; (c) $8 \mathrm{~m}$; (d) $12 \mathrm{~m}$;

TABLE II THE DYNAMIC PAREMETERS VS. DIFFERENT DISTANCE

\begin{tabular}{ccccc}
\hline No & $\begin{array}{c}\text { Distance } \\
(\mathrm{m})\end{array}$ & $\begin{array}{c}t_{d} \\
(\mathrm{~ms})\end{array}$ & $\begin{array}{c}\Delta P \\
(\mathrm{MPa})\end{array}$ & $\begin{array}{c}I^{+} \\
(\mathrm{MPa} . \mathrm{ms})\end{array}$ \\
\hline 1 & 3 & 1.343 & 0.80480 & 0.2576 \\
2 & 4 & 1.474 & 0.47780 & 0.2235 \\
\hline
\end{tabular}




\begin{tabular}{lcccc}
\hline 3 & 6 & 4.217 & 0.13420 & 0.1864 \\
4 & 7 & 4.264 & 0.1023 & 0.1532 \\
5 & 8 & 5.040 & 0.08964 & 0.1277 \\
6 & 10 & 5.532 & 0.05342 & 0.0942 \\
7 & 12 & 6.09 & 0.02937 & 0.0788 \\
8 & 14 & 8.190 & 0.02447 & 0.0772 \\
9 & 17 & 7.590 & 0.01588 & 0.0498 \\
10 & 24 & 8.540 & 0.01514 & 0.0428 \\
11 & 27 & 9.430 & 0.01195 & 0.0414 \\
12 & 31 & 10.110 & 0.00918 & 0.0355 \\
\hline
\end{tabular}

The overpressure value and corresponding fitting curve by polynomial fitting and exponential fitting are shown in Fig.15(a) and Fig.15(b), the root mean squared error of which is 0.0223 and 0.0478 , respectively. The fitted function of overpressure are

$\Delta P=-0.1631\left(\frac{2}{R}\right)+2.041\left(\frac{2}{R}\right)^{2}+0.0594\left(\frac{2}{R}\right)^{3}$

and

$\Delta P=0.02671 \mathrm{e}^{\frac{10.342}{R}}$ according to the equation (6) and equation (7) respectively. The results in Fig. 15(c) show the error between the measured overpressure data from LPWON and estimated data from polynomial fitting method, exponential fitting method and wired system. It is shown that the lowest overpressure error $(<5 \%)$ at the nearest distance way from explosion center for all models due to less interference from the ground. The overpressure error of polynomial fitting method has less error than the counterpart of exponential fitting method, showing that the polynomial fitting method is more suitable for overpressure fitting calculation. It is also found the errors of two polynomial fitting method is close to $50 \%$ as the distance is 17 $m$. Thus, as for this experiment, it is reasonable that the error $(<20 \%)$ is valid according to the empirical statistics of shock wave monitoring, as distance ranges from $7 \mathrm{~m}$ to $12 \mathrm{~m}$.

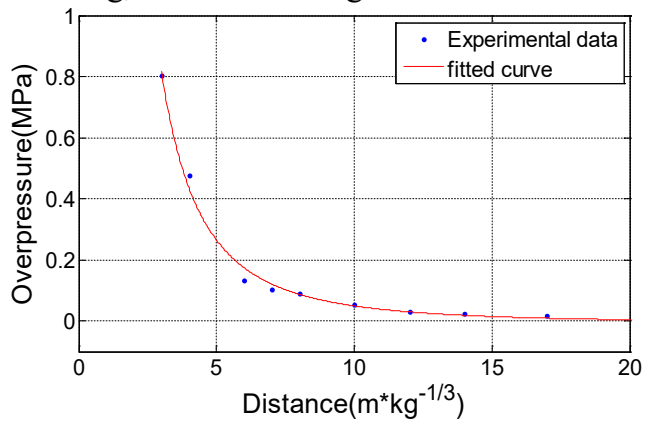

(a)

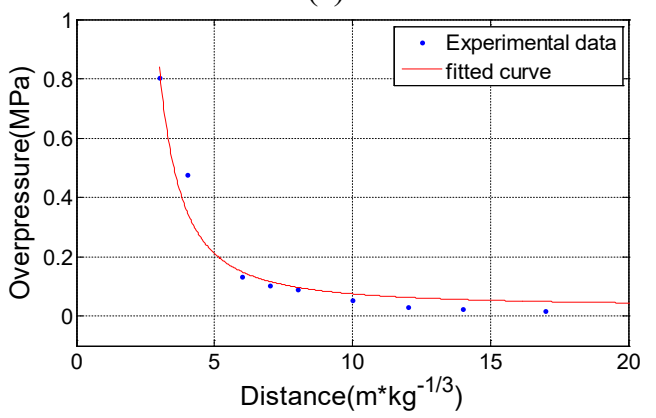

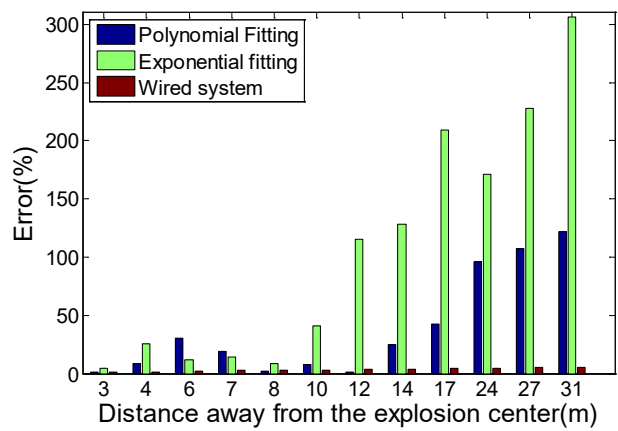

(c)

Fig.15. Overpressure value and corresponding fitted curve in (a) Polynomial fitting method (b) Exponential fitting method. (c) The error between measured value from LPWON vs. pressure assessed in two fitting methods and wired system

Moreover, the duration of the positive phase value and corresponding fitted curve is shown in Fig.16(a), the fitted function is $t_{d}=0.213 R^{0.5824} 8^{0.9103}$ according to the equation (7) with the root mean squared error of 0.7423 . It is noticeable that the error of $t_{d}$ between M.A.Sadoski model and the obtained measured data below $20 \%$ as distance is above $6 \mathrm{~m}$, as shown in Fig.16(b). Fig.17(a) shows that the impulse value and corresponding fitted curve is show in, and the fitted function o is $I^{+}=\frac{0.8968}{R}$ according to the equation (8) with the root mean squared error of 0.0198 . The error of $I^{+}$between Kinney's model and the obtained measured data below 20\% as distance ranges from $3 m$ to $24 m$, as shown in Fig. 17 (b).

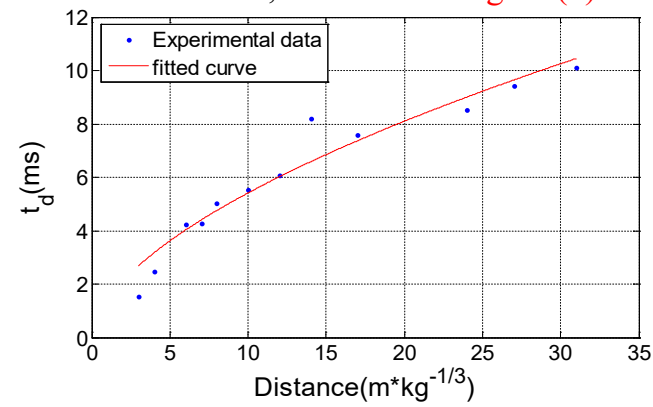

(a)

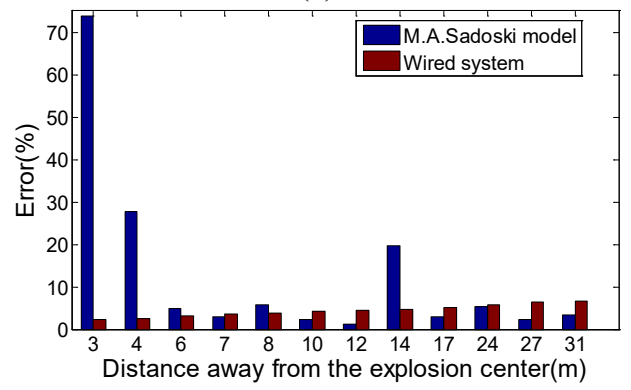

(b)

Fig. 16. (a) The duration of the positive phase value and corresponding fitted curve; (b) The error between measured value from LPWON vs. $t_{d}$ assessed in M.A.Sadoski model and wired system.

The experimental results are prone to many complicate factors such as ground reflection pressure, temperature, humidity, wind velocity, especially the irregularity of TNT explosion. We prove that the correctness and feasibility of LPWON for blast wave monitoring is achieved by the comparison with wired system. More importantly, from above analysis, we can learn 
that the errors of $\Delta P, t_{d}, I^{+}$are credible as the distance ranges from $7 \mathrm{~m}$ to $12 \mathrm{~m}$, which provides an advisable reference for how to deploy monitoring points for a specific experiment implementation.

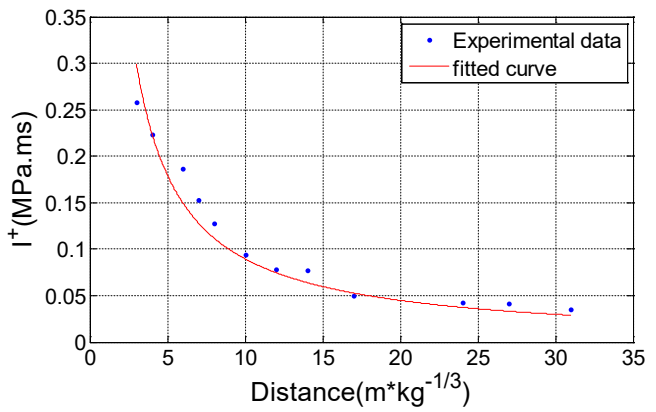

(a)

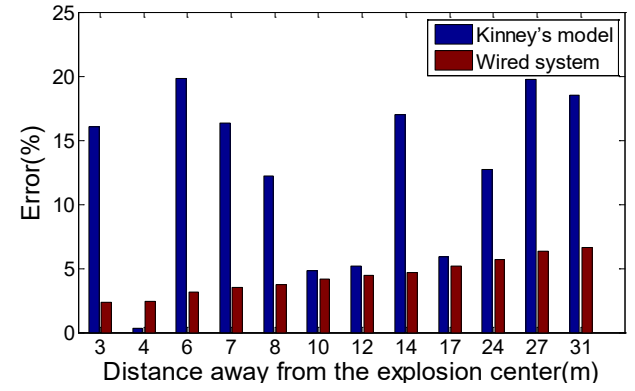

(b)

Fig. 17. (a) The impulse value and corresponding fitted curve; (b) The error between measured value from LPWON vs. $I^{+}$assessed in Kinney's model and wired system

\section{B. Wireless Transmission Quality Evaluation in Air-blast Testing}

To further investigate the network quality performance of the LFWON in wild air-blast monitoring, the LFWONs placed in the LFWON s' line of sight in wild area sends 1000 packets to the base-station, with the highest output power level of 20 $\mathrm{dBm}$. The Fig. 18 demonstrates the relationship between RSSI and PDR in terms of the delivery statistics of packets in the base-station. It is concluded that the average PDR exceeds $86 \%$ with the RSSI higher than $-70 \mathrm{dBm}$, which indicates that the network quality is acceptable at the RSSI setting configuration. In contrast, as the RSSI is lower than $-80 \mathrm{dBm}$, while the average PDR can drop to the lowest value of $5.8 \%$ and the maximum standard deviation of PDR reaches $34.1 \%$. In this respect, the network communication is unreliable, and thus the RSSI value of LFWON should be set above $-70 \mathrm{dBm}$ if a stable communication quality is to be established.

In addition, the data throughput between LFWONs and basestation under different spreading factor ( $\mathrm{SF}$ ) settings $(\mathrm{SF}=6,7$, $8,9,10,11)$ is investigated. Large SF together with higher receiver sensitivity contributes to higher throughput. Fig.19 demonstrates that the throughput vs. different distances is achieved as SF is from 6 to 11 . The throughput is $68.3 \mathrm{Kbps}$ at the distance of $200 \mathrm{~m}$ and descends to $3.9 \mathrm{Kbps}$ at $700 \mathrm{~m}$ as $\mathrm{SF}$ is equal to 6 . In contrast, the throughput can reach 68.3 Kbps shorter than the distance of $400 m$ as SF is equal to 11, which outperforms throughput as SF is equal to 6. In this respect, the throughput remains at $60.3 \mathrm{Kbps}$ as the distance is extended to $500 \mathrm{~m}$. The evaluation proves that the large SF can enlarge the transmission range and strengthen the performance of data delivery.

Any more discussion and reflection?

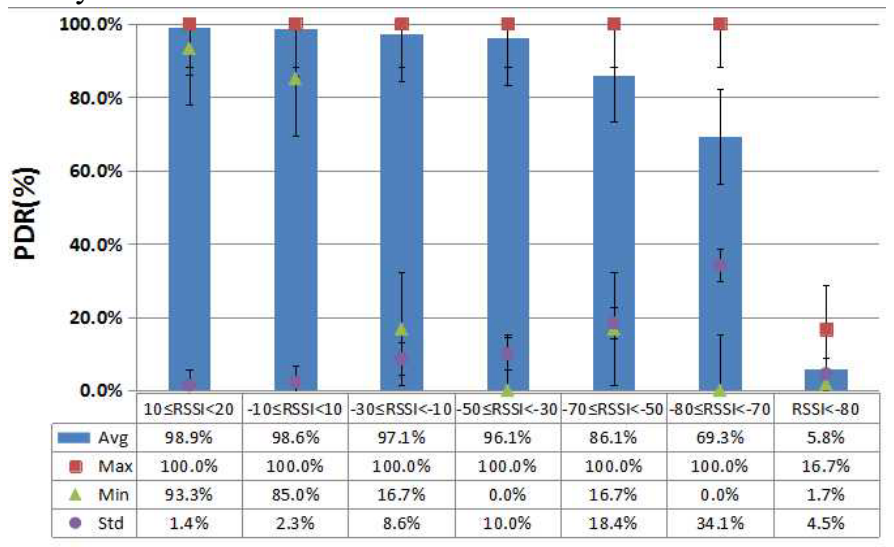

RSSI level(dBm)

Fig. 18. Relationship between PDR vs RSSI

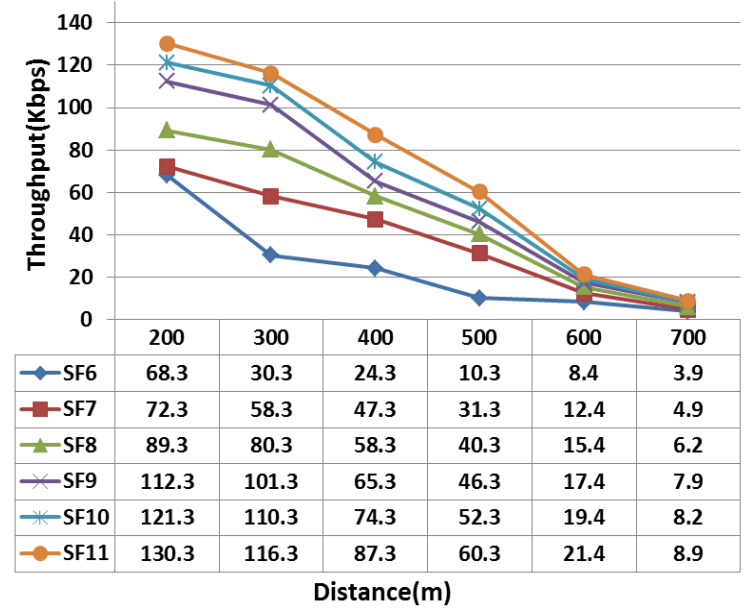

Fig. 19. Relationship between throughput vs distance in different SF

\section{CONCLUSION}

The major contribution of this research proposes a novel a new lightweight underground FPGA-based wireless overpressure node with the resistance to high-temperature and high-pressure environment for SW monitroing. In this approach, the paper reports the implementation of LFWON equipped with XC6SLX9-2TQG144C FPGA, RF transceiver, and Nand flash storage module. Furthermore, the wireless overpressuremonitoring network design method, the installation of piezoelectric pressure transducer, the mechanical model of piezoelectric pressure transducer and the physical structure of LFWON are proposed. The errors of $\Delta P(7 m-12 m), t_{d}(>6$ $m$ ), and $I^{+}(3 m-24 m)$ from proposed LFWON are below 20\% in comparison with wired system, which provides an advisable reference for how to deploy monitoring points for a specific experiment implementation. Finally, it is proved that the RSSI value of LFWON should be set above $-70 \mathrm{dBm}$ for stable communication quality. In future, the approach proposed in this 
paper may shed insight on the development of WSN and IoT technology on explosive blast overpressure monitoring system. Furthermore, we can explore how the proposed node can be extended or applied for high-precision, multi-hop blast overpressure monitoring network and more network scenarios.

\section{REFERENCES}

[1] A. Austin, B. B. Kapil, H. James, et al, "An optical fibre transducer for measuring kinetics of skull-brain interaction in a surrogate model of the human head subjected to blast overpressure," IEEE Sensors Journal, pp.1-1, 2018.

[2] C. L. M. Donald, A. M. Johnson, D. Cooper D, et al, "Detection of BlastRelated Traumatic Brain Injury in U.S. Military Personnel," New England Journal Medicine, vol.364, no.22, pp.860-861, 2011.

[3] V. D. Alphonse, A. R. Kemper, S. M. Duma, "Effects of Filtering on Experimental Blast Overpressure Measurements," Biomedical Sciences Instrumentation, vol. 51, pp.143-150, 2015.

[4] S. Ouellet, M. Phillippens, "The multi-modal responses of a physical head model subjected to various blast exposure conditions," Blast waves, vol. 28, no. 1, pp.19-36, 2018.

[5] W. Fickett, C. William, "Detonation: Theory and Experiment," Dover Publications, 2011

[6] A. Brill, Y. Me-Bar, M. Siman, et al, "Diaphragm gauge for measuring explosive impulse,"International Journal of Impact Engineering, vol. 38, no. (8-9), pp. 765-769, 2011

[7] K. Cheval, O. Loiseau, V. Vala, "Laboratory scale tests for the assessment of solid explosive blast effects. Part I: Free-field test campaign," Journal of Loss Prevention in the Process Industries, vol. 23, no. 5, pp. 613-621, 2010.

[8] L. K. Stewart, B. Durant, J. Wolfson, et al, "Experimentally generated highg shock loads using Hydraulic Blast Simulator," International Journal of Impact Engineering, vol. 69, pp. 86-94, 2014.

[9] H. Y. Grisaro, A. N. Dancygier, "Characteristics of combined blast and fragments loading,"International Journal of Impact Engineering, vol. 116, pp. 51-64, 2018.

[10] N. F. Scilly, "Measurement of the explosive performance of high explosives," Journal of Loss Prevention in the Process Industries, vol.8, no.5, pp.265-273, 1995.

[11] S. Gao, Y. J. Lin, J. J. Zhu, "The Effects of Mounting Structure and Incident Angle on the Measurement of Piezoelectric Pressure Probe Sensors in Free-field Testing," IEEE Sensors Journal, vol. 19, no. 17, pp. 7226-7233, 2019.

[12] S. Gao, Y. G. Tian, X. W. Dai, "A B-Spline Method With AIS optimization for 2-D IoT-Based Overpressure Reconstruction," IEEE Internet of Things, Vol.7, no.3, pp. 2005-2013, 2020.

[13] D. Oshita, S.H.R. Hosseini, K. Mawatari, et al, "Two Successive ShockWaves Generated by Underwater Pulse Electric Discharge for Medical Applications," IEEE Transactions on Plasma Science, vol.42, pp.3209-3214, 2014.

[14] H. Zhou, R. Han, Q. Liu, et al, "Generation of Electrohydraulic Shock Waves by Plasma-Ignited Energetic Materials: II. Influence of Wire Configuration and Stored Energy," IEEE Transactions on Plasma Science, vol.43, pp.4009-4016, 2015.

[15] K. Spranghers, I. Vasilakos, D. Lecompte, et al, "Full-Field Deformation Measurements of Aluminum Plates Under Free Air Blast Loading," Experimental Mechanics, vol. 52, no. 9, pp. 1371-1384, 2012.

[16] C. Fouchier, D. Laboureur, L. Youinou, et al, "Experimental investigation of blast wave propagation in an urban environment," Journal of Loss Prevention in the Process Industries, vol. 49, pp. 248-265, 2017.

[17] H. Zhou, Y. Zhang, R. Han, et al, "Signal Analysis and Waveform Reconstruction of Blast waves Generated by Underwater Electrical Wire Explosions with Piezoelectric Pressure Probes," Sensors, vol. 16, no. 4, pp. 573, 2016.

[18] J. K. Sangjoon, M. G. Gwang, Y. J. Na, et al, "Wireless Ground Reaction Force Sensing System Using a Mechanically Decoupled Two-Dimensional Force Sensor," IEEE/ASME Transactions on Mechatronics, vol. 25, no. 1, pp. 564-571, 2020

[19] L. Cen, S. N. Melkote, J. Castle , et al, "A Wireless Force-Sensing and Model-Based Approach for Enhancement of Machining Accuracy in Robotic Milling," IEEE/ASME Transactions on Mechatronics, vol. 21, no. 5, pp. 22272235, 2016.

[20] J. Gao, G. Yan, "A Novel Power Management Circuit Using a SuperCapacitor Array for Wireless Powered Capsule Robot," IEEE/ASME Transactions on Mechatronics, vol.22, no.3, pp.1444-1455, 2017.
[21] A. S. Kesar, E. Weiss, "Wave Propagation Between Buried Antennas," IEEE transactions Antennas and Propagation, vol. 61, no. 12, pp. 6152-6156, 2013.

[22] J. Fourmann, A. Coustou, H. Aubert, et al, "Wireless pressure measurement in air blast using PVDF sensors," in Proceedings of IEEE sensors, Orlando, United States, 2016.

[23] J. Fourmann, A. Coustou, H. Aubert, et al, "Wireless sensors for the incident pressure measurement in air blast," in Proceedings of 46th EuMC, London, UK, 2017, pp.1339-1342.

[24] P. Schumacher, C, Schuster, A. Jimenez-Saez, et al, "Passive chipless wireless pressure sensor for Harsh and reflective environments," in Proceedings of 11th GeMiC, Freiburg, Germany, 2018.

[25] S. Gao, X. H. Zhang, C. C. Du, et al. "A Multi-Channel Low-Power WideArea Network with High-Accuracy Synchronization Ability for Machine Vibration Monitoring," IEEE Internet of Things, pp.1-8, 2019.

[26] C. N. Kingery, G. Bulmash, Air blast parameters from TNT spherical air burst and hemispherical surface burst. U. S. Army Ballistic Research Laboratory, USA, 1984.

[27] G. Kinney, K. Graham, 1985. Explosive Shocks in Air, second ed. Spring Science Business Media, New York.

[28] J. Riondet, A. Coustou, H. Aubert, et al, "Design of air blast pressure sensors based on miniature silicon membrane and piezoresistive gauges," in $J$. Phys.: Conf. Ser. 922012019, 2017.

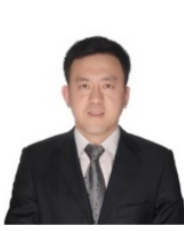

Shang Gao(M'15) received his BEng., MSc., PhD degree in school of measuring and testing technology and instrument engineering from Nanjing University of Aeronautics and Astronautics, Nanjing, China, in 2007, 2010, 2017, respectively. He has also paid a visit to Newcastle University in United Kingdom for the academic research on wireless piezoelectric network from 2015 to 2016. Now he is an assistant professor in the School of Mechanical Engineering, Nanjing University of Science \& Technology, Nanjing, China. His main research area is industrial wireless sensor network, structural health monitoring, smart sensor and measurement system development. He has been selected as a top young talent in the School of Mechanical Engineering in NJUST.(email: shang.gao@njust.edu.cn)

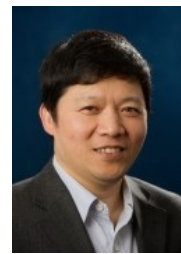

Guiyun Tian (M'01 - SM'03) received the B.Sc.degree in metrology and instrumentation and M.Sc.degree from the University of Sichuan, China, in 1985 and 1988, respectively, and the Ph.D. degree from the University of Derby, Derby, U.K., in 1998. He was a Lecturer, a Senior Lecturer, a Reader, a Professor, and the Head of the Group of Systems Engineering, University of Huddersfield, Huddersfield, UK, from 2000 to 2006. Since 2007, he has been with Newcastle University, Newcastle upon Tyne, U.K., where he is a Chair Professor of sensor technologies. Currently, he is also an adjunct Professor with School of Automation Engineering, University of Electronic Science and Technology of China. (e-mail: g.y.tian@newcastle.ac.uk).

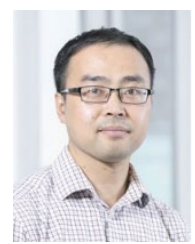

Xuewu Dai (M'09) received his BEng. in Electronic Engineering and MSc. in Computer Science from the Southwest University, China, and $\mathrm{PhD}$ in Electronic and Electrical Engineering from the University of Manchester, UK, in 1999, 2003 and 2008, respectively. He is a senior lecture at the Department of Mathematics, Physics and Electrical Engineering, Northumbria University. His interests include robust state estimation and condition monitoring, wireless sensor actuator networks, networked control systems and industrial Internet of Things.(e-mail: xuewu.dai@northumbria.ac.uk) 
Qing Zhang received the B.Sc. degree in 2012 from North China Electric Power University and received the Ph.D. degree in 2018 from Huazhong University of Science and Technology. She is currently a postdoctor with the College of Automation Engineering, Nanjing University of Aeronautics and Astronautics, Nanjing, China. Her current research interests include electromagnetic nondestructive testing and evaluation, modeling of eddy current fields and computation, and signal processing, and sensors.(e-mail: zhangqing@nuaa.edu.cn)

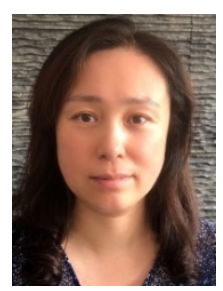

Zhiling Wang received B.S. and M.S. degree from Yanshan University, China, in 2003 and 2006, respectively. The Ph.D. from Nanjing University of Aeronautics and Astronautics, China, in 2017. She is currently an associate professor at the school of Mechanical and Electrical Engineering, Jinling Institute of Technology, Nanjing, China. She main research interests are structural health monitoring, sensor technology, signal processing and information fusion. (e-mail:wzz@jit.edu.cn)

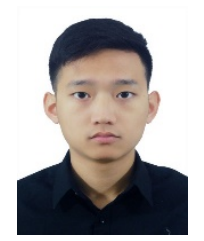

Xinge Yang is a master student in the Department of Mechanical \& Aerospace Engineering, Nanyang Technological University, Singapore. His main research area is wireless sensor network and shock wave monitoring.(e-mail: yxg96@outlook.com)

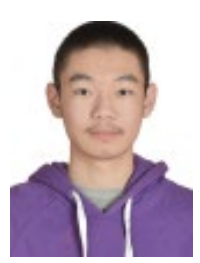

Qiaomu Wang is pursuing the bachelor's degree with the School of Mechanical Engineering, Nanjing University of Science and Technology, Nanjing, His main research area is wireless sensor networks. (email: Qm20180607@njust.edu.cn)

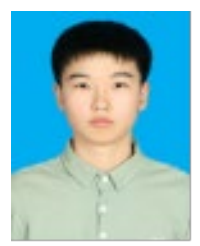

Naishu Jia is pursuing the bachelor's degree with the School of Mechanical Engineering, Nanjing University of Science and Technology, Nanjing, China. Her main research area is wireless sensor networks. (e-mail: 917101170102@njust.edu.cn). 